\title{
Perfil fitoquímico e propriedade antioxidante de diferentes genótipos de frutos do umbuzeiro (Spondias tuberosa Arruda Câmara): uma revisão
}

\author{
Phytochemical profile and antioxidant property of different genotypes of umbuzeiro fruits \\ (Spondias tuberosa Arruda Câmara): a review \\ Perfil fitoquímico y propiedad antioxidante de diferentes genotipos de frutos umbuzeiro (Spondias \\ tuberosa Arruda Câmara): una reseña
}

Recebido: 10/11/2021 | Revisado: 20/11/2021 | Aceito: 25/11/2021 | Publicado: 06/12/2021

\author{
Marcílio Nunes Moreira \\ ORCID: https://orcid.org/0000-0001-5958-8302 \\ Universidade Federal de Sergipe, Brasil \\ E-mail: marciliio10@gmail.com \\ Élia Karina de Carvalho Costa \\ ORCID: https://orcid.org/0000-0002-3749-9277 \\ Instituto Federal de Educação, Ciência e Tecnologia Baiano, Brasil \\ E-mail: elia.costa@ifbaiano.edu.br \\ Sérgio Luiz Rodrigues Donato \\ ORCID: https://orcid.org/0000-0002-7719-4662 \\ Instituto Federal de Educação, Ciência e Tecnologia Baiano, Brasil \\ E-mail: sergio.donato@ifbaiano.edu.br \\ Narendra Narain \\ ORCID: https://orcid.org/0000-0003-3913-5992 \\ Universidade Federal de Sergipe, Brasil \\ E-mail: narendraprocta@gmail.com
}

\begin{abstract}
Resumo
O umbuzeiro, planta pertencente ao gênero das Spondias e família Anacardiaceae, possui 18 espécies que estão localizadas nas Américas, Ásia e Oceania. O fruto tem recebido destaque no mercado nacional e internacional por sua variedade de compostos bioativos, que apresentam efeitos nutracêuticos e terapêuticos. Entretanto, um melhor aproveitamento pode ser obtido através do conhecimento da variabilidade genética desses frutos. Diante disso, objetivou-se realizar uma revisão a respeito da composição bioativa e química como também da atividade antioxidante de frutos de umbu em relação a variação destes parâmetros entre diferentes genótipos do fruto. Apesar das boas propriedades físico-químicas, nutricionais, sensoriais, nutracêuticas e terapêuticas, os frutos do umbuzeiro apresentam comercialização limitada aos locais onde são produzidos devido ao período de sazonalidade, por esta razão, conhecer as características e propriedades específicas de cada genótipo dos frutos do umbuzeiro é de extrema importância, uma vez que, conhecendo tais propriedades, é possível apontar diferentes formas de processamento, melhorias nas técnicas de cultivo, colheita, beneficiamento e processamento e aumento da produtividade, entre outros.

Palavras-chave: Umbuzeiro; Propriedades; Bioativos.
\end{abstract}

\begin{abstract}
The umbu tree, a plant belonging to the Spondias genus and Anacardiaceae family, has 18 species that are located in the Americas, Asia and Oceania. The fruit has been highlighted in the national and international market for its variety of bioactive compounds, which have nutraceutical and therapeutic effects. However, a better use can be obtained through the knowledge of the genetic variability of these fruits. Therefore, the objective was to carry out a review regarding the bioactive and chemical composition as well as the antioxidant activity of umbu fruits in relation to the variation of these parameters between different fruit genotypes. Despite the good physicochemical, nutritional, sensory, nutraceutical and therapeutic properties, the umbuzeiro fruits are marketed limited to the places where they are produced due to the seasonal period, for this reason, to know the specific characteristics and properties of each genotype of the fruits of the umbuzeiro is extremely important, since, knowing these properties, it is possible to point out different forms of processing, improvements in cultivation techniques, harvesting, processing and processing and increased productivity, among others.
\end{abstract}

Keywords: Umbu tree; Properties; Bioactives.

\section{Resumen}

El árbol umbu, planta perteneciente al género Spondias y familia Anacardiaceae, tiene 18 especies que se encuentran ubicadas en América, Asia y Oceanía. La fruta ha sido destacada en el mercado nacional e internacional por su 
variedad de compuestos bioactivos, que tienen efectos nutracéuticos y terapéuticos. Sin embargo, se puede obtener un mejor aprovechamiento mediante el conocimiento de la variabilidad genética de estos frutos. Por tanto, el objetivo fue realizar una revisión sobre la composición bioactiva y química, así como la actividad antioxidante de los frutos de umbu en relación a la variación de estos parámetros entre diferentes genotipos de frutos. A pesar de las buenas propiedades fisicoquímicas, nutricionales, sensoriales, nutracéuticas y terapéuticas, los frutos de umbuzeiro se comercializan limitados a los lugares donde se producen debido al período estacional, por ello, conocer las características y propiedades específicas de cada genotipo de los frutos. del umbuzeiro es sumamente importante, ya que, conociendo estas propiedades, es posible señalar diferentes formas de procesamiento, mejoras en las técnicas de cultivo, recolección, procesamiento y procesamiento y aumento de la productividad, entre otros.

Palabras clave: Árbol umbu; Propriedades; Bioactivos.

\section{Introdução}

Use o parágrafo como modelo (fonte: TNR 10 - justificado - espaço 1,5). O umbu, fruto do umbuzeiro, é uma planta que pertence ao gênero das Spondias, tem seus frutos colhidos de forma extrativistas sendo principal destaque na região nordeste do Brasil se adaptando as condições climáticas da região. Este fruto tem como característica ser uma drupa arredondada; no seu estágio de maturação maduro, tem característica de cor de casca amarelo-esverdeada e cor de polpa branco-esverdeada sendo quase aquosa suculenta, com sabor característico (Folegatti et al., 2003; Lima Filho, 2011). É rico em vitamina $\mathrm{C}$ e amplamente consumido na forma in natura e processado pela população local, por seus atributos sensoriais, sabor doce acidulado, bem como, por seu aroma e sabor característicos (Vidigal et al., 2011; de Souza et al., 2021).

As plantas do umbuzeiro em um único período de colheita anual, a depender das condições climáticas ao qual se encontram, são capazes de produzir até 300kg de fruto por planta (Lima Filho, 2011). A produção brasileira no ano de 2018 foi de 7762 t com a maioria da sua produção se concentrando na região nordeste do Brasil e tendo o estado da Bahia como líder na produção desse fruto (IBGE, 2019). O umbuzeiro é resistente a seca e seus frutos são fontes de renda para maioria dos agricultores familiares da região semiárida. No entanto, sua safra é limitada e possui alta perecibilidade, com tempo de vida útil de dois a três dias após a colheita (de Souza et al., 2021).

Os frutos de umbu têm ganhado interesse de mercado nacional e internacional recentemente, apresentando grande potencial comercial, por se apresentarem como fonte de compostos bioativos (Almeida et al., 2011; Silva et al., 2012), tendo grande importância nutricional e terapêutica. Entretanto, esses frutos ainda são pouco explorados, sendo escassa a quantidade de informações e estudos acerca da variabilidade nas características genéticas desses frutos (Zeraik et al., 2016; Rufino et al., 2010).

Zielinski (2014) estudando a quantidade de compostos fenólicos, carotenoides e atividade antioxidante in vitro de polpa de frutas congeladas consumidas no Brasil encontrou para o fruto do umbu valores de compostos fenólicos de 158,54 (mg GAE/kg), flavonoides 69,30 (mg CTE/kg) e os seguintes carotenoides: $\beta$-caroteno 4,16 (mg de $\beta$-caroteno/100 g) e licopeno 5,67(mg/100g), comprovando a presença de compostos bioativos e da atividade antioxidante in vitro pelos métodos de DPPH e FRAP.

São poucos os trabalhos na literatura que realizam análises entre diferentes genótipos do fruto do umbuzeiro. Nesse contexto, objetivou-se com essa pesquisa explanar sobre os estudos de caracterização de frutos do umbuzeiro, de diferentes genótipos, principalmente em relação a sua capacidade antioxidante e composição química e avaliar as possíveis diferenças entre as variações genéticas, no intuito de identificar o genótipo com maior teor de compostos benéficos à saúde, levando a possibilidade de, através deste estudo, novos estudos surgirem no intuito de , indicar determinado genótipo ao processamento e de modo a se obter produtos diversificados com um maior valor nutricional agregado. 


\section{Metodologia}

\subsection{Tipo de estudo}

A presente pesquisa trata-se de uma revisão bibliográfica exploratória e integrativa de literatura, de abordagem qualitativa. De acordo com Cyriaco et al. (2017), o método qualitativo tem o intuito de descrever os estudos através da observação, contextualização e análise lógica dos dados levantados, afim de responder as questões levantadas e obter uma conclusão coesa.

\subsection{Local da pesquisa}

O presente trabalho trata-se de uma revisão de literatura que teve como fundamento pesquisas a busca por pesquisas bibliográficas, realizadas nas bases de dados científicos como Science Direct, Scopus, Wiley, Online Library e Scielo, além de artigos presentes na Biblioteca Virtual do Google Acadêmico. Utilizou-se os termos: "umbu", "bioactive compounds", "umbu genotypes", "antioxidants in umbu", "characterization of umbu fruit", "umbu quality", "total phenolics", "umbu carotenoids" e "antioxidant activity of umbu".

\subsection{Período do estudo}

O período de busca nas bases eletrônicas, bem como, nos documentos utilizados para a pesquisa foi de janeiro a março de 2021.

\subsection{Critério de inclusão e exclusão}

Como critérios de inclusão e embasamento, utilizou-se: Artigos indexados, informes agropecuários, anais de congresso, entre outros, em Língua Portuguesa e Língua Inglesa e texto completo, com recorte temporal de 10 anos, ou seja, entre 2011 a 2021. Foram excluídos os artigos repetidos ou que não estavam relacionados ao tema proposto. Diante do exposto, foram selecionadas 33 (trinta e três) publicações que se adequam ao tema: o perfil fitoquímico e atividade antioxidante do umbu.

\section{Resultados e Discussão}

Após a leitura minuciosa do conteúdo selecionado para embasamento desta pesquisa, visando os principais estudos que se adequam ao tema de propriedades fitoquímicas e antioxidantes dos frutos do umbuzeiro, selecionou-se 33 (trinta e três) publicações e suas principais características (Autores, ano, título, periódico e fator de impacto), na qual, estão descritas na Tabela 1. 
Tabela 1 - Descrição das características das publicações selecionadas para pesquisa.

\begin{tabular}{|c|c|c|c|}
\hline Autores & Título & Periódico & $\begin{array}{l}\text { Fator de } \\
\text { impacto }\end{array}$ \\
\hline Almeida et al., 2011 & $\begin{array}{l}\text { Bioactive compounds and antioxidant activity of fresh } \\
\text { exotic fruits from northeastern Brazil }\end{array}$ & Food Research International & 6.475 \\
\hline Barros et al., 2021 & Elaboração de Suco Tropical de Umbu. & $\begin{array}{lll}\text { Research, } & \text { Society and } \\
\text { Development } & & \\
\end{array}$ & 1.78 \\
\hline Barroso et al., 2019 & $\begin{array}{l}\text { Phytochemical profile of genotypes of Euterpe edulis } \\
\text { Martius-Juçara palm fruits }\end{array}$ & & \\
\hline Bhargav et al., 2018 & $\begin{array}{l}\text { Diversity of flavonoids profile in China aster } \\
\text { [Callistephus chinensis (L.) Nees.] genotypes }\end{array}$ & Industrial Crops and Products & 5.645 \\
\hline Chen et al., 2021 & $\begin{array}{l}\text { Bioactive compounds and fruit quality of Chinese } \\
\text { raspberry, Rubus chingii Hu varied with genotype and } \\
\text { phenological phase }\end{array}$ & Scientia Horticulturae & 3.463 \\
\hline de Assis et al., 2020 & $\begin{array}{l}\text { Determination of water-soluble vitamins and } \\
\text { carotenoids in Brazilian tropical fruits by High } \\
\text { Performance Liquid Chromatography }\end{array}$ & Heliyon & 2.85 \\
\hline de Lima et al., 2015 & $\begin{array}{l}\text { Caracterização de frutos de genótipos selecionados de } \\
\text { umbu-cajazeira (Spondias sp.) }\end{array}$ & Interciencia & 0.396 \\
\hline de Menezes et al., 2017 & $\begin{array}{l}\text { Influence of the maturation stage on the physical- } \\
\text { chemical quality of fruits of umbu (Spondias tuberosa). }\end{array}$ & Scientia Agropecuaria & 2.137 \\
\hline de Sousa Araújo et al., 2008 & $\begin{array}{l}\text { A new approach to study medicinal plants with tannins } \\
\text { and flavonoids contents from the local knowledge }\end{array}$ & Journal of Ethnopharmacology & 4.360 \\
\hline de Souza et al., 2021 & $\begin{array}{l}\text { Secagem da polpa do umbu (Spondias tuberosa) em } \\
\text { camada de espuma. }\end{array}$ & $\begin{array}{l}\text { Research, } \\
\text { Development }\end{array}$ & 1.78 \\
\hline Dias et al., 2019 & $\begin{array}{l}\text { Extraction of umbu (Spondias tuberosa) seed oil using } \\
\mathrm{CO} 2 \text {, ultrasound and conventional methods: } \\
\text { Evaluations of composition profiles and antioxidant } \\
\text { activities }\end{array}$ & $\begin{array}{l}\text { The Journal of Supercritical } \\
\text { Fluids }\end{array}$ & 4.577 \\
\hline Dutra et al., 2017 & $\begin{array}{l}\text { Bioaccessibility and antioxidant activity of phenolic } \\
\text { compounds in frozen pulps of Brazilian exotic fruits } \\
\text { exposed to simulated gastrointestinal conditions }\end{array}$ & Food Research International & 6.475 \\
\hline dos Santos Neto et al., 2021 & $\begin{array}{l}\text { Licor funcional a base de umbu (Spondias tuberosa } \\
\text { Arruda) e microalga (Spirulina spp.). }\end{array}$ & $\begin{array}{lll}\text { Research, } & \text { Society } & \text { and } \\
\text { Development } & & \\
\end{array}$ & 1.78 \\
\hline Gomes et al., 2020 & $\begin{array}{l}\text { Germplasm of Brazilian winter squash (Cucurbita } \\
\text { moschata D.) displays vast genetic variability, allowing } \\
\text { identification of promising genotypes for agro- } \\
\text { morphological traits }\end{array}$ & bioRxiv & - \\
\hline GOUVÊA et al., 2017 & $\begin{array}{l}\text { Effect of enzymatic treatment on the rheological } \\
\text { behavior and vitamin } \mathrm{C} \text { content of Spondias tuberosa } \\
\text { (umbu) pulp }\end{array}$ & $\begin{array}{l}\text { Journal of food science and } \\
\text { technology }\end{array}$ & 2.701 \\
\hline Granato et al., 2018 & $\begin{array}{l}\text { Antioxidant activity, total phenolics and flavonoids } \\
\text { contents: Should we ban in vitro screening methods? }\end{array}$ & Food chemistry & 7.514 \\
\hline Gundogdu et al., 2017 & $\begin{array}{l}\text { Phenolic compounds, bioactive content and antioxidant } \\
\text { capacity of the fruits of mulberry (Morus spp.) } \\
\text { germplasm in Turkey }\end{array}$ & Folia Horticulturae & 1.873 \\
\hline Islam et al., 2013 & $\begin{array}{l}\text { A comparative study of the antioxidant, antimicrobial, } \\
\text { cytotoxic and thrombolytic potential of the fruits and } \\
\text { leaves of Spondias dulcis. }\end{array}$ & $\begin{array}{l}\text { Asian Pacific journal of tropical } \\
\text { biomedicine }\end{array}$ & 1.545 \\
\hline Melo e Andrade (2010), & $\begin{array}{l}\text { Compostos bioativos e potencial antioxidante de frutos } \\
\text { do umbuzeiro Bioactive compounds and antioxidant } \\
\text { potential from the "umbuzeiro" fruits }\end{array}$ & Alimentos e Nutrição Araraquara & - \\
\hline Nayab et al., 2020 & $\begin{array}{l}\text { Genotypes and harvest maturity influence the } \\
\text { nutritional fruit quality of mulberry }\end{array}$ & Scientia Horticulturae & 3.463 \\
\hline
\end{tabular}




\begin{tabular}{|c|c|c|c|}
\hline Neto et al., 2013 & 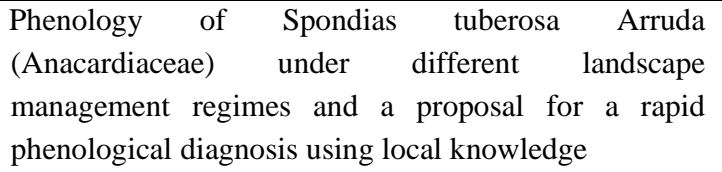 & $\begin{array}{l}\text { Journal of Ethnobiology and } \\
\text { Ethnomedicine }\end{array}$ & 3.007 \\
\hline Ribeiro et al., 2019 & $\begin{array}{l}\text { Nutrients and bioactive compounds of pulp, peel and } \\
\text { seed from umbu fruit }\end{array}$ & Ciência Rural & 0.803 \\
\hline Rui et al., 2012 & $\begin{array}{l}\text { A validated solid-phase extraction HPLC method for } \\
\text { the simultaneous determination of gallic acid, catechin } \\
\text { and epicatechin in rhubarb decoction }\end{array}$ & $\begin{array}{l}\text { Chinese Journal of Natural } \\
\text { Medicines }\end{array}$ & 3.000 \\
\hline Schiassi et al., 2018 & $\begin{array}{l}\text { Fruits from the Brazilian Cerrado region: Physico- } \\
\text { chemical characterization, bioactive compounds, } \\
\text { antioxidant activities, and sensory evaluation }\end{array}$ & Food chemistry & 7.514 \\
\hline Schweiggert e Carle, 2017 & $\begin{array}{l}\text { Carotenoid deposition in plant and animal foods and its } \\
\text { impact on bioavailability. }\end{array}$ & $\begin{array}{l}\text { Critical Reviews in Food Science } \\
\text { and Nutrition }\end{array}$ & 11.176 \\
\hline Silva et al., 2012 & $\begin{array}{l}\text { Bioactive compounds and antioxidant activity in fruits } \\
\text { of clone and ungrafted genotypes of yellow mombin } \\
\text { tree. }\end{array}$ & Food Science and Technology & 4.952 \\
\hline Taleon et al., 2012 & $\begin{array}{l}\text { Effect of genotype and environment on flavonoid } \\
\text { concentration and profile of black sorghum grains }\end{array}$ & Journal of Cereal Science & 3.616 \\
\hline Thassya er al., 2019 & $\begin{array}{l}\text { UPLC-MS-ESI-QTOF analysis and antifungal activity } \\
\text { of the Spondias tuberosa Arruda leaf and root } \\
\text { hydroalcoholic extracts }\end{array}$ & Antibiotics & 4.639 \\
\hline Vidigal et al., 2011 & $\begin{array}{l}\text { Effect of a health claim on consumer acceptance of } \\
\text { exotic Brazilian fruit juices: Açaí (Euterpe oleracea } \\
\text { Mart.), Camu-camu (Myrciaria dubia), Cajá (Spondias } \\
\text { lutea L.) and Umbu (Spondias tuberosa Arruda). }\end{array}$ & Food Research International & 6.475 \\
\hline Yaldiz; Camlica, 2019 & $\begin{array}{l}\text { Variation in the fruit phytochemical and mineral } \\
\text { composition, and phenolic content and antioxidant } \\
\text { activity of the fruit extracts of different fennel } \\
\text { (Foeniculum vulgare L.) genotypes }\end{array}$ & Industrial Crops and Products & 5.645 \\
\hline Zeraik et al., 2016 & $\begin{array}{l}\text { Antioxidants, quinone reductase inducers and } \\
\text { acetylcholinesterase inhibitors from Spondias tuberosa } \\
\text { fruits. }\end{array}$ & Journal of Functional foods & 4.451 \\
\hline Zielinski et al., 2014 & $\begin{array}{l}\text { The association between chromaticity, phenolics, } \\
\text { carotenoids, and in vitro antioxidant activity of frozen } \\
\text { fruit pulp in Brazil: an application of chemometrics. }\end{array}$ & Journal of Food Science & 2.701 \\
\hline Zielinski et al., 2014 & $\begin{array}{l}\text { Chemical composition, sensory properties, provenance, } \\
\text { and bioactivity of fruit juices as assessed by } \\
\text { chemometrics: a critical review and guideline. }\end{array}$ & $\begin{array}{l}\text { Comprehensive reviews in food } \\
\text { science and food safety }\end{array}$ & 12.24 \\
\hline
\end{tabular}

Fonte: Autores.

\subsection{Umbuzeiro}

O umbuzeiro (Spondias tuberosa), árvore frutífera, comumente encontrada no bioma caatinga, de forma nativa, especificamente na região semiárida do nordeste e em algumas regiões do sudeste do Brasil. As árvores dessa espécie costumam nascer a partir da polinização de insetos, o que caracteriza sua forma nativa. Tornando-se de extrema importância para o bioma, visto que, é utilizado por polinizadores e dispersores durante a estação seca, período em que ocorre a frutificação dessa espécie (Gouvêa et al., 2017; Neto et al., 2013).

Tendo papel de destaque na região nordeste do Brasil, essas árvores são frutíferas, em sua grande maioria, exploradas através do extrativismo; dentre essas, estão as espécies: Spondias mombin L. (cajazeira), Spondias purpurea L. (cirigueleira), Spondias cytherea Sonn. (cajaraneira), Spondias tuberosa Arr. Câm. (umbuzeiro) e Spondias spp. (umbucajazeira e umbugueleira) (Martins \& Melo, 2005). Por sua vez a espécie Spondias tuberosa Arruda Camara (umbuzeiro) se destaca por 
grande importância social, comercial e ecológica sendo endêmica da caatinga do Brasil, com ocorrência natural no cerrado, mata atlântica e região pré-Amazônica (Drumond et aI., 2016; De Araújo, 2016; Santos, 1998).

Sendo uma xerófita que perdura por mais de 30 anos, com altura média em torno de 6 metros e podendo atingir até 15 metros de diâmetro, sendo uma alternativa de proteção aos animais e ao solo, durante as horas mais quentes do dia. (Duque, 2004; Santos, Nascimento e Oliveira, 1999; Pires, 1990; Pires e Oliveira, 1986). Com um sistema radicular que pode atingir até um metro de profundidade e longas raízes laterais, com caules subterrâneos, denominados de xilopódios, os quais são responsáveis por realizar o controle de respiração da árvore, podendo armazenar água e nutrientes como proteína, fibra bruta, lipídeos, tanino, amido, enxofre, fósforo, cálcio e magnésio (Lima, 1996), sendo vitais para nutrição vegetal e sua sobrevivência durante o período de estiagem, garantindo a sobrevivência dessas plantas por mais de trinta anos (Duque, 2004). Tais características apresentadas anteriormente permitem ao umbuzeiro crescer em regiões com temperaturas na faixa de $12{ }^{\circ} \mathrm{C}$ a $38^{\circ} \mathrm{C}$, umidade relativa (UR) do ar de $30 \%$ a $90 \%$, exposição solar de 2.000 - 3.000 horas/luz/ano e volume de chuva de 400 a 800mm entre os meses de novembro a fevereiro, se adaptando a locais com até 1600mm/ano (Saturnino 2011; Duque, 2004).

Tendo o período de frutificação com início 25 dias após o florescimento e levando em torno de 120 dias para atingir a sua maturação dos frutos do umbuzeiro, possuindo somente um período de colheita por ano, período este cujo clima e ambiente tem total influencia, tornando possível uma colheita de até 300kg de fruto por safra, de uma única planta (LIMA, 2011). Os frutos do umbuzeiro foram descritos por Folegatti et al. (2003). Lima Filho (2011) cita o fruto como uma drupa de forma arredondada a ovalada, liso ou com algumas protuberâncias, coloração de casca amarelo-esverdeado, polpa branco-esverdeada mole e suculenta quase aquosa com sabor característico.

Devido ao sabor agridoce sendo suave característico, os frutos de umbu são bastante apreciados, caracterizados como sendo um fruto exótico e diferenciado, principalmente nas regiões de produção, onde os frutos beneficiam a agricultura familiar de pequena escala, como sendo fonte de renda de muitos agricultores, que comercializam o fruto in natura e processado sob a forma de suco, geleia, sorvete e diversas sobremesas. Contudo, apesar das tentativas de pequenos produtores de aproveitarem os frutos do umbu, a quantidade de fruto desperdiçada, todos os anos, é alta, visto que, ainda não foram desenvolvidas tecnologias para o processamento de toda a produção, bem como, há a falta de recomendação de frutos nativos locais, como fonte de vitaminas à população, de modo a estimular o consumo, produção e a expansão do comércio destes (de Assis et al., 2020; Gouvêa et al., 2017).

\subsection{Características do fruto (umbu)}

\subsubsection{Características Físico-químicas do umbu}

Características físicas, como formato, tamanho e sabor, são determinantes para a qualidade de frutos. Esses atributos físicos aliados à composição química dos frutos promovem a aceitabilidade dos frutos pelos consumidores (Gondim et al., 2013; Lima et al., 2002).

Apresentando sabor e aromas característicos e rico em vitamina C, os frutos de umbu se destacam como frutos de boa aparência e qualidade nutritiva, sua composição permite que os frutos possam ser apreciados na forma in natura, em polpa e processada (Vidigal et al., 2011; Neves e Carvalho, 2005; Carvalho et al., 2008).

Entre os caracteres físicos do fruto, de Menezes et al. (2017), relataram que a média encontrada para vitamina C foi 14,78mg de ácido ascórbico/100g de polpa e variações encontradas para sólidos solúveis (SS) foram de 9,54 a $13,74{ }^{\circ}$ Brix e 7,83 a 10,77 para a relação SS/AT. Contudo, segundo de Lima et al. (2015), valores de diâmetro e vitamina C em frutos de umbu apresentaram divergência entre diferentes genótipos estudados. Os autores concluíram também que os frutos de umbu de diferentes genótipos sofreram influência nos valores de $\mathrm{pH}$, relação de sólidos solúveis/acidez titulável e açúcar total.

Santos et al. (2017), afirmam que não há variação dos valores de pH de frutos de umbu em estádios de maturação 
verde e intermediário, embora a acidez seja maior em frutos verdes, e tende a diminuir conforme o processo de amadurecimento avança, devido aos processos respiratórios dos frutos. Em relação ao teor de sólidos solúveis, o estudo ainda afirma que, o teor de sólidos solúveis nos frutos de umbu tendem a aumentar durante o amadurecimento, devido ao aumento no teor de açúcares, consequência da hidrólise de amido e pectina. O teor de SS é uma importante variável, estando associada ao teor de açúcares contido no fruto e, juntamente com a acidez titulável, associada ao ponto de maturação adequado do fruto (Ferreira et al., 2015).

O umbu se apresenta como fonte de vitamina C sendo encontrado também em sua composição elementos como cálcio, ferro e fosforo que se tornam essências para uma boa dieta, como expressado na Tabela 2.

Tabela 2. Composição nutricional (por 100 gramas de parte comestível) do umbu.

\begin{tabular}{cc}
\hline & Composição por 100 gramas da parte comestível \\
\hline Calorias & $37 \mathrm{kcal}$ \\
Proteína & $0,80 \mathrm{~g}$ \\
Carboidratos & $9,40 \mathrm{~g}$ \\
Fibra Alimentar & $2,0 \mathrm{~g}$ \\
Cálcio & $12,0 \mathrm{mg}$ \\
Magnésio & $0,03 \mathrm{mg}$ \\
Fósforo & $13,0 \mathrm{mg}$ \\
Ferro & $0,10 \mathrm{mg}$ \\
Potássio & $152 \mathrm{mg}$ \\
Cobre & $0,04 \mathrm{mg}$ \\
Zinco & $0,40 \mathrm{mg}$ \\
Tiamina & $0,05 \mathrm{mg}$ \\
Riboflavina & $0,06 \mathrm{mg}$ \\
Piridoxina & $0,03 \mathrm{mg}$ \\
Vitamina C & $24,10 \mathrm{mg}$ \\
\hline
\end{tabular}

Fonte: NEPA (2011) \& IBGE (2011).

De acordo com estudos realizados por dos Santos Neto et al. (2021), o fato dos frutos do umbu apresentarem excelentes características físico-químicas com teores de sólidos solúveis, pH e acidez titulável, o seu processamento industrial, armazenamento e conservação são alternativas viáveis e promissoras.

\subsubsection{Compostos bioativos do umbu}

As frutas podem se apresentar como uma excelente fonte de compostos bioativos, além de ser uma fonte renovável, apresentando em sua composição compostos fenólicos, vitaminas, carotenoides e minerais, alguns compostos desses bioativos obtêm propriedades antioxidantes (Schiassi et al., 2018).

Em estudo realizado por Barros et al. (2021) foi demonstrado que apesar do umbu ser um fruto pouco explorado, ele é rico em compostos bioativos e nutrientes fundamentais para a saúde, e por ter excelentes propriedades, pode ser amplamente utilizado no desenvolvimento de produtos alimentícios, como sucos, permitindo a praticidade e aumento da forma de consumo deste fruto para os consumidores.

Estes compostos fitoquímicos compreendem uma extensa quantidade de substâncias que incluem flavonoides e não flavonoides, que em sua maioria são compostos de metabolismo secundários encontrados nas plantas, que por sua vez os 
utilizam como forma de proteção para a suas células contra danos que podem ser causados por radicais livres, com a sua propriedade de oxirredução eles se tornam muito importantes para proteção das moléculas em seus compartimentos celulares contra oxidação (Orsavová et al., 2019). Os compostos fenólicos também estão associados a características dos alimentos tais como cor, sabor em diversas plantas. (Chirinos et al., 2009).

Segundo Dias et al. (2019) e Sameh et al. (2018), membros do gênero Spondias são comumente utilizados na medicina tradicional para tratamento de muitas doenças, por apresentarem substâncias antioxidantes, protetoras de úlceras, hepatoprotetoras, fotoprotetoras, anti-inflamatórias, antiartrítico, antidemência, antipirético, analgésicas, trombolíticas, hipoglicêmicas, antifertilidade, anti-hipertensivas, antimicrobianas, antivirais e anti-helmínticas, relacionadas à elevada gama de fitoconstituintes presentes neste gênero, podendo assim destacar a presença de taninos, flavonoides, esteróis, óleos essenciais, polissacarídeos e aminoácidos.

\subsubsection{Fenólicos Totais do umbu}

De acordo com Dias et al. (2019) e Dutra et al. (2017), os compostos fenólicos, constituídos por anéis aromáticos e grupos hidroxilas, podem ser encontrados em quantidades elevadas em frutos nativos e exóticos, e têm ganhado destaque recentemente, por apresentarem propriedades importantes, a exemplo da atividade antioxidante.

Podendo ser divididos em ácidos fenólicos, flavonoides, antocianinas e taninos, a literatura tem relatado a presença desses compostos em frutos de umbu, conforme relatado por Dutra et al. (2017), ao estudarem a atividade antioxidante e quantidade de compostos fenólicos em polpas de frutas e polpas congeladas, que identificaram o perfil fenólico de polpa de umbu, tendo encontrado um alto teor de fenólicos livres totais, predominantemente o ácido protocatecuico. Santos et al. (2019), ao estudarem extratos hidroalcoólicos de raízes e folhas de umbu, encontraram alcalóides, esteróides, fenóis, flavonoides, triterpenóides e xantonas e afirmaram a presença de fenóis e flavonoides em todos os extratos estudados enquanto Ribeiro et al. (2019), ao estudarem os compostos bioativos da polpa, casca e semente do umbu, citaram um teor de 1.775 mg GAE.100 g-1 encontrado na casca do umbu e $1.746 \mathrm{mg}$ GAE.100 g $\mathrm{g}^{-1}$ encontrado na polpa comercial, além da presença dos compostos rutina e quercetina.

\subsubsection{Teor de Carotenóides no umbu}

Os carotenoides são compostos associados a cor dos alimentos que podem variar do amarelo ao vermelho, podendo ser encontrados amplamente em alimentos de origem animal e vegetal, pode trazer diversos benefícios a saúde humana sua bioatividade e dada pelo metabolismo de carotenoides chamados de precursores da vitamina A, que por sua vez e responsável nos seres humanos e mamíferos em geral em manter o crescimento e robustez de celular e pelo sistema visual. O consumo de alimentos ricos em carotenoides é associado a prevenção do envelhecimento prematuro da pele, efeitos benéficos sobre a funcionalidade do sistema imunológico e com sua forte atividade antioxidante, podendo agir na prevenção de doenças crônicas (Schweiggert e Carle, 2017).

Frutas de coloração amarela e laranja são comumente relacionadas a elevados teores de carotenoides, tiamina, ácido fólico, ácido nicotínico, ácido pantotênico, riboflavina, cianocobalamina, piridoxina e biotina (Assis et al., 2020). Ribeiro et al. (2019), estudando compostos bioativos em cascas e sementes de umbu, encontraram um teor de $4632 \mu \mathrm{g} .100 \mathrm{~g}^{-1} \mathrm{de}$ carotenoides; ainda segundo os autores, o teor de carotenoides encontrado no estudo foi maior quando comparado ao teor de carotenoides em camu-camu, mangaba e polpas de caju. O mesmo estudo ainda relatou a presença de $\alpha$-caroteno e $\beta$-caroteno em todas amostras avaliadas, tendo o $\beta$-caroteno se apresentado em maior concentração em todas as amostras avaliadas, para a polpa fresca $80 \mu \mathrm{g} .100 \mathrm{~g}^{-1}$, polpa comercial $140 \mu \mathrm{g} .100 \mathrm{~g}^{-1}$ e casca $1.100 \mu \mathrm{g} .100 \mathrm{~g}^{-1}$. Melo e Andrade (2010), estudando o teor de compostos bioativos e o potencial antioxidantes de frutos do umbuzeiro, ao compararem o teor de carotenoides em frutos de 
umbu em diferentes estágios de maturação, concluíram que o fruto de umbu maduro apresentou valor maior, quando comparado ao fruto semi-maduro, com teores de 3,02 e $1,70 \mu \mathrm{g} .100 \mathrm{~g}^{-1}$.

\subsubsection{Teor de flavonóides no umbu}

Derivados da 2-fenilcromona, sintetizados da fenilalanina, com esqueleto de carbono C6eC3eC6, os flavonoides são uma classe de metabólitos secundários encontrados em plantas e fungos, identificado dentre mais de 5000 compostos classificados em flavonoides, flavanonas, flavonas, isoflavonas, flavonóis e antocianinas (Bhargav et al., 2018), sendo conhecidos por serem compostos de baixo peso molecular e por apresentarem estrutura que favorece o sequestro de radicais livres, sendo portanto, antioxidantes mais efetivos que as vitaminas C e E (Alves et al., 2007; Song et al., 2012). Segundo Taleon et al. (2012), flavononas são as classes de flavonoides comumente encontradas em altas concentrações em frutos de genótipos amarelos.

Os frutos e a casca do umbuzeiro (S. tuberosa) estão principalmente associados a efeitos anti-inflamatórios e cicatrizantes, devido à presença de flavonoides (de Sousa Araújo et al., 2008). Quanto ao teor de flavonoides em frutos de umbu, Rufino et al. (2010) relataram um teor de $6,9 \mathrm{mg} .100 \mathrm{~g}^{-1}$; a literatura relata dois grupos de flavonoides encontrados em frutos de umbu, rutina e quercetina, como compostos em maior quantidade, com predominância da quercetina $\left(9 \mathrm{mg} .100 \mathrm{~g}^{-1}\right)$ (Santos et al., 2019).

Todos esses estudos avaliaram flavonoides como um grupo coletivo, mas o uso de compostos específicos é importante quando são considerados para uso como nutracêutico ou corante.

\subsubsection{Atividade Antioxidante}

A atividade antioxidante é um dos parâmetros utilizados para se avaliar o valor nutricional de frutas. Todavia com a variedade de cultivares de frutas e consequentemente a grande gama de composição química, os dados sobre atividade antioxidante se tornam inconsistentes, consequentemente dificultando o estabelecimento de padrões e de resultados mais precisos a partir dos dados disponíveis na literatura. Cada fruta dispõe de várias especificidades na atividade antioxidante dos diversos compostos bioativos encontrados nas respectivas frutas, essas especificidades se estendem em relação aos diferentes genótipos de uma mesma fruta. Com diferentes métodos analíticos de avaliação de atividade antioxidante, os resultados obtidos podem oferecer uma visão mais ampla das interações entre os fatores, como a genótipos da fruta estudada, que podem influenciar na atividade antioxidante, levando a um maior conhecimento das propriedades antioxidantes de distintas genótipos (Soobrattee et al., 2005; Granato et al., 2018).

Sameh et al. (2018), relacionam a elevada atividade antioxidante de plantas do gênero Spondias à presença de flavonoides e fenólicos. Em estudo, Ribeiro et al. (2019) avaliando a composição nutricional e compostos bioativos polpa do fruto do umbu, com intuito de descobrir seu potencial de aplicação na indústria e comparação com uma polpa congelada, já comercializada. Os frutos foram coletados na cidade de Milagres, Bahia para elaboração da polpa fresca e a polpa congelada adquirida de uma pequena empresa na cidade de Itamari, Bahia. Os valores encontrados para atividade antioxidante determinada pelo método de redução do ABTS, para a polpa congelada adquirida comercialmente e para polpa fresca foram de 105,24 ( $\left.\mu \mathrm{mol} \mathrm{TE} . \mathrm{g}^{-1}\right)$ e 25,24 $\left(\mu \mathrm{mol}\right.$ TE. $\left.\mathrm{g}^{-1}\right)$, respectivamente. Através dos resultados obtidos pode se perceber a diferença entre a constituição química das polpas de diferentes locais, os autores não detalham informações como genótipo ou condições climáticas e ambientais ao qual os frutos foram cultivados e posteriormente adquiridos. Através da discrepância de resultados se faz necessária maiores informações sobre a capacidade antioxidante de polpas do fruto do umbu afim de se definir um genótipo superior para utilização na indústria.

Zeraik et al. (2016), utilizando frutos de S. tuberosa Arr. Cam, sendo coletados na cidade de João Pessoa no estado da 
Paraíba, realizaram diferentes ensaios de atividade antioxidante; dentre eles, utilizaram o método de atividade sequestrante do radical livre $\mathrm{DPPH}^{*}$, encontrando em seu trabalho $89 \%$ de inibição, do radical livre resultados estes encontrados no extrato metanoico da polpa de umbu. Islam et al. (2013), realizaram um estudo comparativo dos antioxidantes, antimicrobianos, citotóxicos e potencial trombolítico dos frutos e folhas de Spondias dulcis, frutos esse conhecido popularmente como cajámanga, utilizando a o método de redução do DPPH e extrato metanoico da fruta em questão encontraram valores menores em comparação ao controle positivo que foi usado no trabalho (ácido L- ascórbico), sendo os valores de $\mathrm{IC}_{50}(1,91 \mu \mathrm{g} / \mathrm{mL})$ e de $\mathrm{IC}_{50}(4,94 \mu \mathrm{g} / \mathrm{mL})$, respectivamente. Vale ressaltar que apesar de serem frutos diferentes, o umbu e o cajá-manga pertencem ao mesmo gênero das Spondias, entretanto não são encontrados na literatura, trabalhos que desenvolvem os estudos em frutos das mesmas espécies cuja única variação entre si seja o genótipo.

Zielinski et al. (2014), utilizando a metodologia de potencial antioxidante redutor férrico (FRAP) analisaram polpa de umbu congelada e de outros frutos do mesmo gênero como cajá e seriguela ao qual também são de destaque na região nordeste e compartilham de características semelhantes ao umbu, com os valores da capacidade antioxidante em FRAP de 14.613,33 $\mu \mathrm{mol} \mathrm{TE} / \mathrm{kg}, 12.644,44 \mu \mathrm{mol} \mathrm{TE} / \mathrm{kg}$ e 11.542,22 $\mu \mathrm{mol} \mathrm{TE} / \mathrm{kg}$ sendo seriguela, cajá e umbu, respectivamente, ficando evidente a presença de atividade antioxidante no gênero das Spondias quando na espécie do umbu. É de suma importância que haja mais pesquisa voltadas ao gênero das Spondias, e em especifico a espécie do umbu tendo em vista que até o presente momento são desconhecidos trabalhos, que expressam a diferenciação de genótipos em suas pesquisas.

Rufino et al. (2010), avaliando o potencial antioxidante de 18 frutas tropicais brasileiras, dentre elas estavam presentes o cajá e o umbu, utilizando o ensaio ORAC, encontrou para o cajá 92,7\% e para o umbu 63,4\% de inibição de oxidação, valores estes encontrados em polpas frescas dos frutos. Entretanto, não são encontrados na literatura muitos trabalhos que utilizassem essa metodologia para atividade antioxidante em umbu.

Vale ressaltar, conforme Fang, Meng e Min (2018), que descrevem o consumo de frutas sendo ricas em propriedades antioxidantes podem atuar na preve.nção de doenças como o diabetes, hipertensão, insuficiência cardíaca, AVC, câncer, mal de Alzheimer, reumatismo, esclerose múltipla, artrite deformante, despertando assim o interesse e se descobrir frutas ricas nas propriedades citadas anteriormente a fim de se trazer benefícios a saúde humana.

\subsection{Influência do genótipo na composição nutricional e bioativa de frutos}

Frutos cultivados em um mesmo local e sob as mesmas condições ambientais, podem apresentar diferenças em termos de rendimento, qualidade física e nutricional (Yaldiz; Camlica, 2019). A literatura cita diferença entre valores de sólidos solúveis totais, teor de ácido ascórbico, relação sólidos solúveis/acidez total titulável, índice de amadurecimento, pH, conteúdo de fenólicos e atividade antioxidante devido à diversidade genética existente em um mesmo plantio (Gundogdu et al., 2017; Nayab et al., 2020).

Conforme relatado por Chen et al. (2021), os conteúdos de antocianinas variaram 3 vezes entre dez genótipos de Rubus chingii. Os níveis de terpenois, vitamina C, coloração e firmeza em frutos de dois genótipos foram significativamente diferentes dos demais, apresentando os maiores valores. Um único genótipo se destacou dos demais em relação ao teor de sólidos solúveis e acidez titulável, tendo sido o fruto com melhor sabor. Assim sendo, o que permitiu inferir que diferentes composições químicas foram obtidas de diferentes genótipos de $R$. chingii, o que permite uma seleção entre os genótipos com melhores qualidades de frutas.

Gomes et al. (2020), estudando a influência de genótipos em frutos de Cucurbita moschata, concluíram que os frutos apresentavam diferenças morfológicas, além de diferença em relação a produção, número e massa das sementes dos frutos avaliados e diferenças no teor de carotenoides na polpa dos frutos. Concluindo que correlações genéticas podem fornecer maior produtividade em termos de maior número de frutos por planta, altura e diâmetro destes. 
Diferentes composições de polifenóis, flavonoides, antocianinas e consequente diferença em termos de atividade antioxidante foram encontrados entre seis genótipos de E. edulis por Barroso et al. (2019). Os autores concluíram que dentre os frutos avaliados um genótipo apresentou destaque dentre os demais em termos de atividade antioxidante e compostos bioativos, sugerindo que este fruto deveria ser incluído na dieta diária e que mais estudos deveriam ser realizados para compreender o efeito do consumo do fruto, em específico do genótipo de destaque, na saúde humana.

No jardim clonal implantado na EPAMIG - Empresa de Pesquisa Agropecuária de Minas Gerais há relato de 32 genótipos de umbu, adquiridas em diferentes cidades. Em estudo, foi identificado frutos de 32,98 gramas a 87,19 gramas de peso de fruto sendo as médias obtidas com amostragem de 20 frutos por genótipos (Saturnino, 2011). Tal informação é de suma importância, tendo em vista que autores como Duque (2004) relatou que o peso de frutos de umbu varia de 10 a 20 gramas por frutos; Santos (1997) apresenta informações de que o peso médio dos frutos se encontra na faixa de 15,80 a 26,70 gramas; a EMBRAPA-Empresa Brasileira de Pesquisa Agropecuária indica que no seu banco ativo de germoplasma de umbu da sua unidade Embrapa semiárido localizada em Petrolina, Pernambuco são encontrados valores de peso de fruto que vão de 2,50 a 85,00 gramas, sendo este por sua vez composto por 79 acessos diferentes (Machado e tal, 2015). Vale ressaltar também que esses conflitos de informações indicam os avanços genéticos que vem acontecendo ao longo dos anos.

Se encontram no Instituto Federal de Educação, Ciência e Tecnologia Baiano - Campus de Guanambi diversos acessos de umbuzeiro, mantidos em condições de campo, características climáticas e ambientais únicas da região, com a área implantada no ano de 2007, o campus dispõe de uma área experimental composta por 24 acessos de umbuzeiro, sendo uma parceria entre o Instituto, Embrapa Mandioca e Fruticultura, Cruz das Almas - BA e a Empresa de Pesquisa Agropecuária de Minas Gerais - EPAMIG Norte, Nova Porteirinha - MG.

Essas informações acerca da composição bioativa podem ajudar na seleção de genótipos de maior produtividade e qualidade nutricional, a literatura cita diversos trabalhos investigando a influência do genótipo em diferentes frutos, no entanto, a variação nos constituintes nutricionais de diferentes genótipos em frutos do umbuzeiro no sudoeste baiano permanece desconhecido, assim a caracterização dos frutos se torna de suma importância com relação ao descobrimento de características especificas de cada genótipo, podendo se tornar de grande ferramenta para destiná-los a suas diversas formas de processamento, como relatado anteriormente.

\section{Conclusão}

De acordo com os estudos analisados observou que o umbu é um fruto que apresenta excelentes propriedades nutricionais, químicas, bioativas, fitoquímicas e antioxidantes, no entanto, o estudo dessas características ainda é escasso na literatura, principalmente no que diz respeito à diferenciação e comparação dessas propriedades entre os genótipos.

Haja vista que a sua comercialização ocorre de forma limitada aos locais onde os frutos são produzidos, conhecer as características específicas de cada genótipo é de suma importância e torna-se grande ferramenta para direcionar os frutos a diversas formas de processamento, uma vez que, para o mercado interno, por exemplo, os frutos do umbu que são obtidos exclusivamente de forma extrativista, agrega valor comercial, especialmente para as agroindústrias.

Estudos futuros que viabilizam a caracterização e utilização de cada tipo de genótipo do umbu, e considerados superiores em suas características bioativas e capacidade antioxidantes, são necessários, no intuito de apontar esses genótipos com características específicas a determinado tipo de processamento e desenvolvimento de novos produtos, bem como, propor melhorias em técnicas de manejo, cultivo, colheita, processamento, conservação e aumento da produtividade. 


\section{Referências}

Almeida, M. M. B., de Sousa, P. H. M., Arriaga, Â. M. C., do Prado, G. M., de Carvalho Magalhães, C. E., Maia, G. A., \& de Lemos, T. L. G. (2011). Bioactive compounds and antioxidant activity of fresh exotic fruits from northeastern Brazil. Food Research International, 44(7), $2155-2159$.

Alves, C. Q., Brandão, H. N., David, J. M., David, J. P., \& Lima, L. D. S. (2007). Avaliação da atividade antioxidante de flavonóides. Diálogos \& Ciência, 12, $1-8$.

Barros, A. C., Bispo, H. G. A. R., Reis, P. M. C. L., de Oliveira Vilar, S. B., Junior, N. D. M. A., Oliveira, I. P., \& de Figueiredo, D. V. P. (2021). Elaboração de Suco Tropical de Umbu. Research, Society and Development, 10(5), e17310513048-e17310513048.

Barroso, M. E., Oliveira, B. G., Pimentel, E. F., Pereira, P. M., Ruas, F. G., Andrade, T. U., ... \& Endringer, D. C. (2019). Phytochemical profile of genotypes of Euterpe edulis Martius-Juçara palm fruits. Food Research International, 116, 985-993.

Bhargav, V., Kumar, R., Shivashankara, K. S., Rao, T. M., Dhananjaya, M. V., Sane, A., ... \& Roy, T. K. (2018). Diversity of flavonoids profile in China aster [Callistephus chinensis (L.) Nees.] genotypes. Industrial Crops and Products, 111, 513-519.

Chen, Z., Jiang, J., Li, X., Xie, Y., Jin, Z., Wang, X., ... \& Yang, W. (2021). Bioactive compounds and fruit quality of Chinese raspberry, Rubus chingii Hu varied with genotype and phenological phase. Scientia Horticulturae, 281, 109951.

Da-Costa-Rocha, I., Bonnlaender, B., Sievers, H., Pischel, I., \& Heinrich, M. (2014). Hibiscus sabdariffa L.-A phytochemical and pharmacological review. Food chemistry, 165, 424-443.

Araujo, F. P., Aidar, S. D. T., da Matta, V. M., Monteiro, R., \& de Melo, N. F. (2016). Umbuzeiro: alternativas de manejo sustentável e aproveitamento em áreas de produtores familiares em Uauá, BA. Embrapa Semiárido-Capítulo em livro técnico (INFOTECA-E).

Assis, R. C., Soares, R. D. L. G., Siqueira, A. C. P., de Rosso, V. V., de Sousa, P. H. M., Mendes, A. E. P., ... \& Maia, C. S. C. (2020). Determination of water-soluble vitamins and carotenoids in Brazilian tropical fruits by High Performance Liquid Chromatography. Heliyon, 6(10), e05307.

Lima, M. S. S., Dantas, A. C. V. L., Fonseca, A. A. O., \& Barroso, J. P. (2015). Caracterização de frutos de genótipos selecionados de umbu-cajazeira (Spondias sp.). Interciencia, 40(5), 311-316

Menezes, P. H. S., de Souza, A. A., da Silva, E. S., de Medeiros, R. D., Barbosa, N. C., \& Soria, D. G. (2017). Influence of the maturation stage on the physical-chemical quality of fruits of umbu (Spondias tuberosa). Scientia Agropecuaria, 8(1), 73-78.

Sousa Araújo, T. A., Alencar, N. L., de Amorim, E. L. C., \& de Albuquerque, U. P. (2008). A new approach to study medicinal plants with tannins and flavonoids contents from the local knowledge. Journal of ethnopharmacology, 120(1), 72-80.

Souza, L. M. R., Canuto, M. F. C. S., Silva, D. R. S., Farias, F. P. M., \& dos Santos, F. S. (2021). Secagem da polpa do umbu (Spondias tuberosa) em camada de espuma. Research, Society and Development, 10(11), e488101119955-e488101119955.

Dias, J. L., Mazzutti, S., de Souza, J. A., Ferreira, S. R., Soares, L. A., Stragevitch, L., \& Danielski, L. (2019). Extraction of umbu (Spondias tuberosa) seed oil using CO2, ultrasound and conventional methods: Evaluations of composition profiles and antioxidant activities. The Journal of Supercritical Fluids, 145, 1018 .

Santos Neto, J. P., dos Santos, Y. M. P., Cunha, P. S. J., do Moraes Gatti, V. C., da Silva Barata, H., Nunes, G. G. T., ... \& de Santana, M. C. C. B. (2021). Licor funcional a base de umbu (Spondias tuberosa Arruda) e microalga (Spirulina spp.). Research, Society and Development, 10(3), e42010313557e42010313557.

Duque, G. O Nordeste e as lavouras xerófilas. $4^{\mathrm{a} E d}$. Fortaleza: Banco do Nordeste do Brasil, 2004. 330p.

Dutra, R. L. T., Dantas, A. M., de Araújo Marques, D., Batista, J. D. F., de Albuquerque Meireles, B. R. L., de Magalhães Cordeiro, Â. M. T., ... \& Borges, G. D. S. C. (2017). Bioaccessibility and antioxidant activity of phenolic compounds in frozen pulps of Brazilian exotic fruits exposed to simulated gastrointestinal conditions. Food Research International, 100, 650-657.

Folegatti, M. I., Matsuura, F. C., Cardoso, R. L., Machado, S. S., Rocha, A. S., \& Lima, R. R. (2003). Aproveitamento industrial do umbu: processamento de geléia e compota. Ciência e Agrotecnologia, 27, 1308-1314.

Gomes, R. S., Júnior, R. M., de Almeida, C. F., Chagas, R. R., de Oliveira, R. L., Delazari, F. T., \& da Silva, D. J. H. (2020). Germplasm of Brazilian winter squash (Cucurbita moschata D.) displays vast genetic variability, allowing identification of promising genotypes for agro-morphological traits. bioRxiv.

Gouvêa, R. F., Ribeiro, L. O., Souza, E. F., Penha, E. M., Matta, V. M., \& Freitas, S. P. (2017). Effect of enzymatic treatment on the rheological behavior and vitamin C content of Spondias tuberosa (umbu) pulp. Journal of food science and technology, 54(7), 2176-2180.

Granato, D., Shahidi, F., Wrolstad, R., Kilmartin, P., Melton, L. D., Hidalgo, F. J., ... \& Finglas, P. (2018). Antioxidant activity, total phenolics and flavonoids contents: Should we ban in vitro screening methods?. Food chemistry, 264, 471-475.

Gundogdu, M., Canan, I., Gecer, M. K., Kan, T., \& Ercisli, S. (2017). Phenolic compounds, bioactive content and antioxidant capacity of the fruits of mulberry (Morus spp.) germplasm in Turkey. Folia Horticulturae, 29(2), 251-262.

IBGE, Produção da Extração Vegetal e Silvicultura 2018. Rio de Janeiro: IBGE, 2019.

Islam, S. M. A., Ahmed, K. T., Manik, M. K., Wahid, M. A., \& Kamal, C. S. I. (2013). A comparative study of the antioxidant, antimicrobial, cytotoxic and thrombolytic potential of the fruits and leaves of Spondias dulcis. Asian Pacific journal of tropical biomedicine, 3(9), 682-691.

Lima Filho, J. M. P. (2011). Ecofisiologia do umbuzeiro (Spondias tuberosa, Arr. Cam.). Embrapa Semiárido-Documentos (INFOTECA-E). 
Lima, M., Resende, G. D., \& Brito, N. D. (1996). Colletotrichum gloeosporioides causando lesoes em frutos de umbu (Spondias tuberosa) na regiao do submedio Sao Francisco. In Embrapa Semiárido-Resumo em anais de congresso (ALICE). Horticultura Brasileira, Brasília, DF, v. 14 , n. 1, p. 93, 1996.

Lucas, C. D. J.; Anunciene, B. Physico-chemical characterization of fruits of umbuzeiro (Spondias tuberosa Arr . Câm .) from Janaúba-MG. 2015.

Machado, C. D. F., de Oliveira, V. R., Maia, M., Alves, R., Ritzinger, R., Soares Filho, W. D. S., \& Fonseca, N. (2015). Banco ativo de germoplasma de spondias da Embrapa. Embrapa Mandioca e Fruticultura-Fôlder/Folheto/Cartilha (INFOTECA-E).

Melo, E. D. A., \& Andrade, R. D. S. (2010). Compostos bioativos e potencial antioxidante de frutos do umbuzeiro Bioactive compounds and antioxidant potential from the "umbuzeiro" fruits. Alimentos e Nutrição Araraquara, 21(3), 453-458.

Nayab, S., Razzaq, K., Ullah, S., Rajwana, I. A., Amin, M., Faried, H. N., ... \& Naz, A. (2020). Genotypes and harvest maturity influence the nutritional fruit quality of mulberry. Scientia Horticulturae, 266, 109311.

NEPA - Núcleo de estudos e pesquisas em alimentação -Tabela Brasileira de Composição de Alimentos. 4. ed. Campinas: UNICAMP, 2011.

Neto, E. M. L., Almeida, A. L., Peroni, N., Castro, C. C., \& Albuquerque, U. P. (2013). Phenology of Spondias tuberosa Arruda (Anacardiaceae) under different landscape management regimes and a proposal for a rapid phenological diagnosis using local knowledge. Journal of Ethnobiology and Ethnomedicine, 9(1), 1-13.

Pires, I. E., \& de Oliveira, V. R. (1986). Estrutura floral e sistema reprodutivo de umbuzeiro. Embrapa Semiárido-Comunicado Técnico (INFOTECA-E).

Pires, M. D. G. D. M. (1990). Estudo taxonomico e area de ocorrencia de Spondias tuberosa Arr. Cam.(umbuzeiro) no Estado de Pernambuco-Brasil. UFRPE.

Ribeiro, L. D. O., Viana, E. D. S., Godoy, R. L. D. O., Freitas, S. C. D., Freitas, S. P., \& Matta, V. M. D. (2019). Nutrients and bioactive compounds of pulp, peel and seed from umbu fruit. Ciência Rural, 49.

Rufino, M. D. S. M., Alves, R. E., de Brito, E. S., de Morais, S. M., Sampaio, C. D. G., Pérez-Jimenez, J., \& Saura-Calixto, F. D. (2007). Metodologia científica: determinação da atividade antioxidante total em frutas pela captura do radical livre DPPH. Embrapa Agroindústria Tropical-Comunicado Técnico (INFOTECA-E).

Rui, S. O. N. G., Cheng, Y., Yuan, T. I. A. N., \& Zhang, Z. J. (2012). A validated solid-phase extraction HPLC method for the simultaneous determination of gallic acid, catechin and epicatechin in rhubarb decoction. Chinese Journal of Natural Medicines, 10(4), 275-278.

Santos, C. A. F., \& de Souza Nascimento, C. E. (1998). Relação entre caracteres quantitativos do umbuzeiro (Spondias tuberosa A. Camara). Pesquisa agropecuaria brasileira, 33(4), 449-456.

Santos, C. A. F., Nascimento, C. D. S., \& de Oliveira, M. C. (1999). Recursos geneticos do umbuzeiro: preservacao, utilizacao e abordagem metodologica. In: Queiroz, MA de; Goedert, CO; Ramos, SRR (Ed.). Recursos genéticos e melhoramento de plantas para o Nordeste brasileiro. Petrolina: Embrapa Semi-Árido; Brasília, DF: Embrapa Recursos Genéticos e Biotecnologia, 1999.

Saturnino, H. M., \& Gonçalves, N. P. (2011). Spondias: umbu, caja-manga and hog plum caja. Informe Agropecuario, $32(264)$, 101-113.

Schiassi, M. C. E. V., de Souza, V. R., Lago, A. M. T., Campos, L. G., \& Queiroz, F. (2018). Fruits from the Brazilian Cerrado region: Physico-chemical characterization, bioactive compounds, antioxidant activities, and sensory evaluation. Food chemistry, 245, 305-311.

Schweiggert, R. M., \& Carle, R. (2017). Carotenoid deposition in plant and animal foods and its impact on bioavailability. Critical Reviews in Food Science and Nutrition, 57(9), 1807-1830.

Silva, F. V. G. D., Silva, S. D. M., Silva, G. C. D., Mendonça, R. M. N., Alves, R. E., \& Dantas, A. L. (2012). Bioactive compounds and antioxidant activity in fruits of clone and ungrafted genotypes of yellow mombin tree. Food Science and Technology, 32, 685-691.

Soobrattee, M. A., Neergheen, V. S., Luximon-Ramma, A., Aruoma, O. I., \& Bahorun, T. (2005). Phenolics as potential antioxidant therapeutic agents: mechanism and actions. Mutation Research/Fundamental and Molecular mechanisms of mutagenesis, 579(1-2), $200-213$.

Taleon, V., Dykes, L., Rooney, W. L., \& Rooney, L. W. (2012). Effect of genotype and environment on flavonoid concentration and profile of black sorghum grains. Journal of Cereal Science, 56(2), 470-475.

Thassya Lucas dos Santos, A., Pereira Carneiro, J. N., Pereira da Cruz, R., Lima Sales, D., Cosmo Andrade, J., de Oliveira Almeida, W., ... \& Coutinho, H. D. M. (2019). UPLC-MS-ESI-QTOF analysis and antifungal activity of the Spondias tuberosa Arruda leaf and root hydroalcoholic extracts. Antibiotics, 8(4), 240.

Vidigal, M. C., Minim, V. P., Carvalho, N. B., Milagres, M. P., \& Gonçalves, A. C. (2011). Effect of a health claim on consumer acceptance of exotic Brazilian fruit juices: Açaí (Euterpe oleracea Mart.), Camu-camu (Myrciaria dubia), Cajá (Spondias lutea L.) and Umbu (Spondias tuberosa Arruda). Food Research International, 44(7), 1988-1996.

Yaldiz, G., \& Camlica, M. (2019). Variation in the fruit phytochemical and mineral composition, and phenolic content and antioxidant activity of the fruit extracts of different fennel (Foeniculum vulgare L.) genotypes. Industrial Crops and Products, 142, 111852.

Zeraik, M. L., Queiroz, E. F., Marcourt, L., Ciclet, O., Castro-Gamboa, I., Silva, D. H. S., ... \& Wolfender, J. L. (2016). Antioxidants, quinone reductase inducers and acetylcholinesterase inhibitors from Spondias tuberosa fruits. Journal of Functional foods, 21, 396-405.

Zielinski, A. A. F., Ávila, S., Ito, V., Nogueira, A., Wosiacki, G., \& Haminiuk, C. W. I. (2014). The association between chromaticity, phenolics, carotenoids, and in vitro antioxidant activity of frozen fruit pulp in Brazil: an application of chemometrics. Journal of Food Science, 79(4), C510-C516.

Zielinski, A. A., Haminiuk, C. W., Nunes, C. A., Schnitzler, E., van Ruth, S. M., \& Granato, D. (2014). Chemical composition, sensory properties, provenance, and bioactivity of fruit juices as assessed by chemometrics: a critical review and guideline. Comprehensive reviews in food science and food safety, 13(3), 300-316. 